\title{
Validation of novel automatic ultra-wideband radar for sleep apnea detection
}

\author{
Yong Zhou ${ }^{1,2}$, Degui Shu ${ }^{1}$, Hangdi Xu' ${ }^{1}$, Yuanhua Qiu ${ }^{1}$, Pan Zhou ${ }^{1}$, Wenjing Ruan ${ }^{1}$, Guangyue Qin ${ }^{3}$, \\ Joy Jin ${ }^{2}$, Hao Zhu ${ }^{4}$, Kejing Ying ${ }^{1}$, Wenxia Zhang ${ }^{1}$, Enguo Chen ${ }^{1}$ \\ ${ }^{1}$ Respiratory and Critical Care Medicine, Sir Run Run Shaw Hospital, School of Medicine, Zhejiang University, Hangzhou 310000, China; \\ ${ }^{2}$ Thoracic Oncology Program, Department of Surgery, Helen Diller Family Comprehensive Cancer Center, University of California San Francisco, \\ San Francisco, CA, USA; ${ }^{3}$ Respiratory and Critical Care Medicine, Zhejiang Hospital, Hangzhou 310000, China; ${ }^{4}$ Respiratory and Critical Care \\ Medicine, Wuyi Campus, Sir Run Run Shaw Hospital, School of medicine, Zhejiang University, Hangzhou 310000, China \\ Contributions: (I) Conception and design: None; (II) Administrative support: None; (III) Provision of study materials or patients: None; (IV) \\ Collection and assembly of data: H Xu, W Ruan, Y Qiu; (V) Data analysis and interpretation: Y Zhou; (VI) Manuscript writing: All authors; (VII) \\ Final approval of manuscript: All authors. \\ Correspondence to: Dr. Enguo Chen; Wenxia Zhang. Respiratory and Critical Care Medicine, Sir Run Run Shaw Hospital, School of Medicine, \\ Zhejiang University, 3 Eastern Qingchun Road, Hangzhou 310000, China. Email: 3195024@zju.edu.cn; 710880270@qq.com.
}

\begin{abstract}
Background: To validate the accuracy of ultra-wideband (UWB) wireless radar for the screening diagnosis of sleep apnea.

Methods: One hundred and seventy-six qualified participants were successfully recruited. Apnea-hypopnea index (AHI) results from polysomnography (PSG) were reviewed by physicians, while the radar device automatically calculated AHI values with an embedded chip. All results were statistically analyzed.

Results: A UWB radar-based AHI algorithm was successfully developed according to respiratory movement and body motion signals. Of all 176 participants, 63 exhibited normal results $(\mathrm{AHI}<5 / \mathrm{hr})$ and the remaining 113 were diagnosed with obstructive sleep apnea. Significant correlation was detected between radar AHI and PSG AHI (Intraclass correlation coefficient 0.98, $\mathrm{P}<0.001$ ). Receiver operating characteristic curve (ROC) analysis revealed high sensitivity and specificity. High concordance in participants with varying gender, age, BMI, and PSG AHI was reached.
\end{abstract}

Conclusions: The UWB radar may be a portable, convenient, and reliable device for obstructive sleep apnea screening.

Keywords: Non-contact; ultra-wideband radar; screening; apnea-hypopnea index (AHI)

Submitted Oct 25, 2019. Accepted for publication Feb 17, 2020.

doi: $10.21037 /$ jtd.2020.02.59

View this article at: http://dx.doi.org/10.21037/jtd.2020.02.59

\section{Introduction}

Sleep apnea is a disease condition that has serious health and financial implications. Obstructive sleep apnea contributes largely. The prevalence of obstructive sleep apnea (OSA), defined at an apnea-hypopnea index (AHI) $\geq 5$, fell at a mean of $22 \%$ (range, $9-37 \%$ ) in men and $17 \%$ (range, $4-50 \%$ ) in women based on a published epidemiological study between 1993 and 2013 (1). It is believed that the prevalence of sleep apnea is still increasing around the world. At present, sleepiness scale evaluation followed by laboratory polysomnography (PSG) is a classic diagnostic procedure for sleep apnea. However, this process has several limitations, including relatively expensive costs, full nighttime occupation of the sleep lab, discomfort due to attached sensors, and long waiting lists. It requires trained technician and physician attendance, and does not allow professionals to monitor patients continuously for long-term or distant follow-up. Furthermore, the most important problem is the fact that a considerable number of sleep apnea patients 
remain undiagnosed and untreated. This underestimated diagnosis is chiefly caused by the lack of public awareness and shortage of sleep centers. Untreated patients may thus experience poor quality of life, adverse cardiovascular events (2). Intermittent hypoxia, intrathoracic pressure changes, arousals, and sleep fragmentation, which occur in association with obstructive sleep apneas, are likely to contribute to increased risk of cardiovascular events through the synergistic combination of heterogeneous mechanisms, including enhanced sympathetic activity, upregulation of the atrial natriuretic peptide and renin-angiotensinaldosterone systems, and recurrent intermittent hypoxiareoxygenation, which resembles the ischemia-reperfusion cycle and favors the release of reactive oxygen species, cytokines, and vasoactive mediators $(3,4)$. Over time, the autonomic and neurohumoral abnormalities perpetuate beyond the offending obstructive events and persist into the daytime, resulting in a disturbance of the overall circadian blood pressure rhythm and an increase in short-and longterm blood pressure variability, which actually promote damage to target organs, including hearth, brain and kidney (5). The impact on the neurocognitive functioning was associated with the blood pressure variability $(6,7)$. Therefore, advances have been made to develop economical and more accessible diagnostic tools, especially those that can be used in patients' homes. However, a recently published review on screening for sleep apnea in adults suggested that there is uncertainty about the accuracy or clinical utility of all potential screening tools (8). The lack of suitable observational methods for large-sample, long-running longitudinal investigations is likely the bottleneck (2).

Recently, a non-contact radar sleep testing technology has emerged. Radar detection integrates radar and biomedical engineering technologies, and can wirelessly penetrate nonmetal substances within certain ranges of distance, detecting signals such as respiration, heartbeat, blood stream, bowel movements, and body motion. The ultra-wideband (UWB) wireless radar system in particular is based on emission and reception of sub-nanosecond pulses, and can provide a low-cost, small-sized, low power consumption and high data rate alternative (9). It is theoretically more sensitive than the traditional belts in detecting respiratory movements. Even the doppler radar can detect thoracic and abdominal movements with high accuracy, up to almost $100 \%$ of sensitivity and specificity. The device accurately detected central sleep apnea, obstructive sleep apnea and mixed sleep apnea (10).
Usually obstructive sleep apnea presents respiratory effort throughout the event. Central sleep apnea shows absence of respiratory effort. And mixed sleep apnea presents absence of respiratory effort at the beginning of the event followed by respiratory effort during the second half. Studies have previously reported that satisfying AHI score values can be approximated from thoracic movements alone (11-15). In addition, body motion sensors such as hand-wearing Jawbone showed good agreement with PSG in measures of total sleep time and wake-after-sleep onset (16). Since radar can detect both respiratory movement and body motion, advantages in sleep apnea syndrome screening and diagnosis are obvious; the technology might be more accurate and can be applied outside sleep centers without trained technician attendance. An algorithm based on certain parameters can classify the severity of sleep apnea syndrome and assess sleep quality (17). However, no large-scale study validating such a promising device in sleep apnea screening has been published thus far. This study adopted simultaneous overnight sleep monitoring with a UWB wireless radar system and PSG, and results were compared to validate the AHI of the UWB wireless radar system.

\section{Methods}

\section{Participants}

The protocol of our study was approved by the Ethics Committee of Sir Run Run Shaw Hospital, and the research was performed in accordance with relevant guidelines. Informed consent for study participation and publication of identifying information/images in an online open-access publication was obtained from all participants. Eligible participants were consecutive patients or healthy volunteers who were referred to sleep labs in Qingchun campus and Xiasha campus of Sir Run Run Shaw Hospital, and Zhejiang Hospital. Patients with unstable medical conditions, such as severe chronic pulmonary disease, severe heart disease, severe obesity (BMI $>45 \mathrm{~kg} / \mathrm{m}^{2}$ ), neuromuscular disorder, inability to apply the diagnostic device or other factors that result in incompletion of the sleep detection, patients unable to sign the study consent were excluded. In total, 176 participants in three hospital sleep centers from September 2015 to February 2017 were recruited with written consent. All the participants were assessed by physicians and detected with PSG and UWB radar simultaneously in the hospitals. There were 125 male and 51 female patients, with a mean age of $38.07 \pm 14.99$, BMI $24.7 \pm 4.24$, and Epworth sleepiness 
Table 1 Demographics of participants

\begin{tabular}{lc}
\hline Item & Total \\
\hline Subjects number & 176 \\
Male sex & 125 \\
Age & $38.1 \pm 15.0$ \\
BMl $\left(\mathrm{kg} / \mathrm{m}^{2}\right)$ & $24.7 \pm 4.2$ \\
Epworth sleepiness scale & $8.8 \pm 1.4$ \\
Total sleep time (min) & $376.7 \pm 89.8$ \\
Oxygen desaturation index of $4 \%(/ \mathrm{h})$ & $32.8 \pm 33.8$ \\
Sleep latency (min) & $27.8 \pm 39.0$ \\
Sleep efficiency index (\%) & $79.0 \pm 15.1$ \\
Arousal index $(/ \mathrm{h})$ & $16.3 \pm 15.1$ \\
\hline
\end{tabular}

scale of $8.8 \pm 1.4$ (Table 1).

\section{Polysomnography and UWB radar sleep screening device}

All participants underwent PSG (Alice5, Respironics Inc, USA) and wireless UWB radar sleep screening device (ZGS01A, Hangzhou Zhaoguan Sensor Technology) detection simultaneously throughout the whole night.

PSG channels included four-channel electroencephalogram (EEG), electro-oculogram (EOG), air-flow at the nose and mouth, submental and leg electromyogram (EMG), electrocardiogram (ECG), chest and abdominal respiratory movement, oxygen saturation (pulse oximetry), a body position sensor, and a snoring microphone. All results were manually interpreted by a sleep technician according to the standard criteria of the '2012 AASM Manual for the Scoring of Sleep and Associated Events' and were subsequently reviewed by certified physicians.

The UWB wireless radar sleep screening device (ZGS01A), a lightweight ball with a diameter of approximately 15 centimeters, was placed on the side table beside the bed 15-25 centimeters above the mattress and about 1.5 meters away from the patient, with its antenna directed towards the patient's chest and abdomen. The patient is free to turn body position during sleep. ZG-S01A has a detection chip inside and connects to an Android app via Bluetooth. The UWB radar, which is within FCC spectrum limitations (6G-8G Hz), can detect both central and obstructive sleep apnea patterns. It begins to work once powered on, and ends once powered off. TST (total sleep time) was calculated in the same fashion as the portable PSG, adjusted based on participant's body motion, respiratory rate and its variation. The device can tell if the patient is in the bed, or body position is changing. The wake time is removed from the total sleep time of the patient during AHI calculation. Radar AHI results were calculated blindly to PSG technician.

\section{Statistical analysis}

Continuous variables have been summarized as mean \pm standard deviation (SD). Intraclass correlation coefficient and Bland-Altman plots were used to assess the correlation and agreement between the PSG AHI and Radar AHI. Kendall tau-b was used to evaluate the concordance of the severity of AHI between PSG and radar results. ROC analysis was adopted to determine the sensitivity and specificity of the radar device. Statistical analysis and graphs were performed with STATA 14.0, SPSS 23.0, and PRISM 7.0. A P value of $<0.05$ was considered to be statistically significant.

\section{Results}

Respiratory movements and body motion signals were collected by radar device simultaneously with PSG monitoring (Figure 1). The radar device was developed and further optimized to improve consistency, and the different types the sleep apnea were detected (Figure 2) (17). In total, 176 participants were recruited to validate this device; all participants successfully completed simultaneous detection through PSG and wireless UWB radar detection. According to AHI criteria, PSG detection revealed 113 sleep apnea syndrome patients, including 26 mild cases, 32 moderate cases, 55 severe cases, and 63 normal cases. The mean PSG AHI was $23.69 \pm 24.63$ events/hr. The wireless radar device revealed 113 sleep apnea syndrome patients, including 25 mild cases, 34 moderate cases, 54 severe cases, and 63 normal cases. The mean radar AHI was $22.66 \pm 24.63$ events/ hr (Table 2).

With a cut-off value of $\mathrm{AHI}=5$, the radar device demonstrated a sensitivity and specificity of $100 \%$ (Table 3). ROC analysis (Figure $3 A$ ) showed the area under the curve was $1.00 \pm 0.00$ ( $95 \%$ confidence interval $1.00-1.00$ ), while the positive predictive value and negative predictive value were both $100 \%$, indicating the high accuracy of the radar 


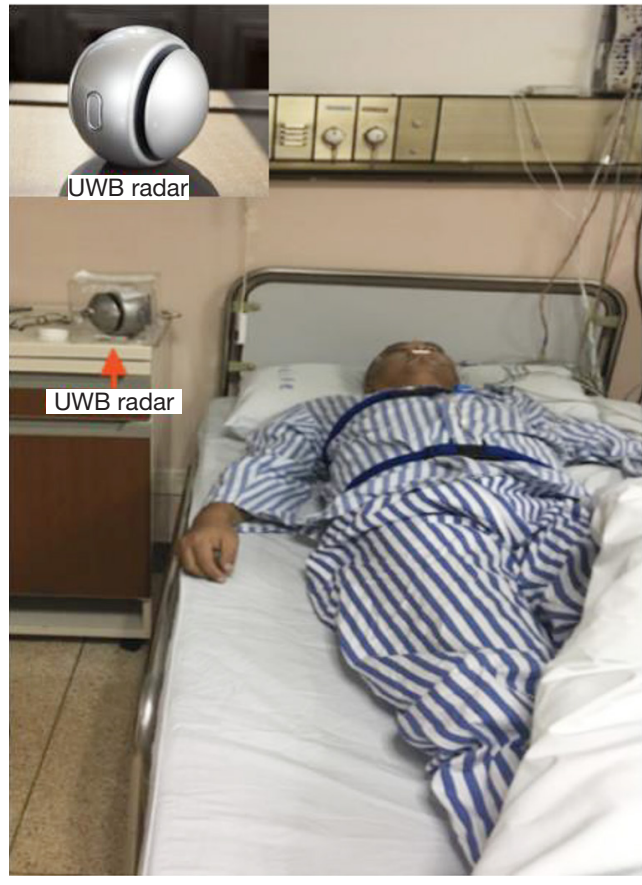

Figure 1 Simultaneous monitoring with the UWB radar and PSG (enlarged UWB radar photo on the left top of the figure). UWB, ultra-wideband; PSG, polysomnography.

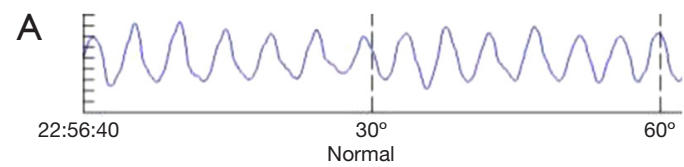

B
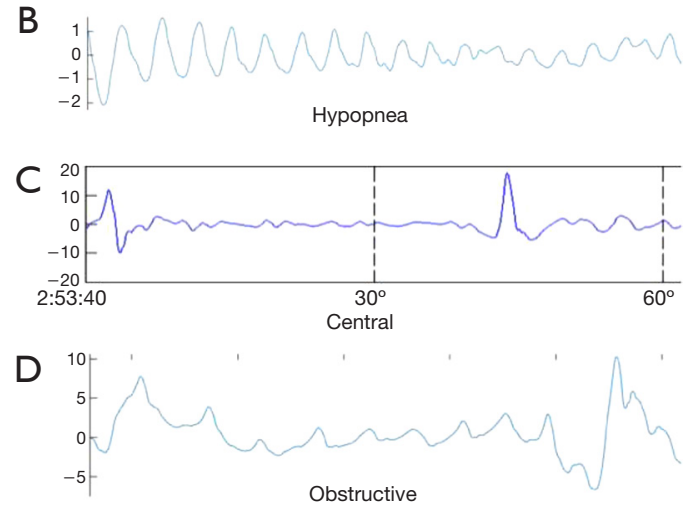

Figure 2 Segments of the radar signal illustrating the scoring of respiratory events. (A) Normal breathing signal; (B) hypopnea showing a slight reduction in the excursion; (C) central apnea showing a complete absence of excursion; (D) obstructive apnea showing a major reduction in the excursion, some excursion appears to still exist, suggesting breathing effort. device in sleep apnea syndrome screening.

With a cut-off value of $\mathrm{AHI}=15$, the radar device demonstrated a sensitivity of $96.55 \%$ and specificity of $95.51 \%$ (Table 3). ROC analysis (Figure 3B) showed the area under the curve was $0.99 \pm 0.005$ ( $95 \%$ confidence interval: $0.98-1.00$ ), with a positive predictive value of $98.9 \%$ and a negative predictive value of $100 \%$.

With a cut-off value of AHI $=30$, the radar device showed a sensitivity of $94.55 \%$ and specificity of $99.17 \%$ (Table 3). ROC analysis (Figure 3C) showed the area under the curve was $0.99 \pm 0.004$ (95\% confidence interval: $0.99-1.00$ ), with a positive predictive value of $98.21 \%$ and a negative predictive value of $98.37 \%$.

Pearson's correlation, Intraclass correlation, and BlandAltman plots each revealed significantly high correlation and good agreement between the radar AHI and PSG AHI (Pearson's $\mathrm{r}=0.97,95 \%$ confidence interval: 0.96-0.98, $\mathrm{P}<0.0001, \mathrm{n}=176$; Intraclass correlation average measure $0.98,95 \%$ confidence interval: $0.98-0.99, \mathrm{P}<0.001$; BlandAltman AHI bias -1.03 , 95\% limits of agreement -13.0410.99) (Figure 3D, Figure $4 A$ ). The radar AHI and PSG AHI agreement was not affected by body mass index (Figure 4B). Both radar AHI and PSG AHI increased consistently with increasing age (Figure $4 C$ ), but radar AHI tended to non-significantly underestimate the severity of OSA with increased age, or PSG AHI (Figure 4D). Subgroup AHI correlation analysis also revealed that both moderate and severe OSA patients had high correlation between radar AHI and PSG AHI, while mild OSA patients did not (Table 2). There was a significantly high concordance of AHI severity (Kendall tau- $\mathrm{b}=0.834, \mathrm{P}<0.0001, \mathrm{n}=176$ ) between PSG and radar results (Table 4).

Bland-Altman plot revealed significantly high correlation and good agreement between radar AHI and PSG AHI both in male and female groups (Figure $5 A, B$ ). PSG AHI was higher in the male group (mean difference $-25.93,95 \%$ confidence interval: $-30.83--21.03, \mathrm{P}<0.0001$ ) (Figure 5C), but radar AHI/PSG AHI agreement between male and female participants showed no statistical difference (mean difference $-0.64,95 \%$ confidence interval: $-2.12-0.84$, $\mathrm{P}=0.39$ ) (Figure 5D). In hypertensive group and normal blood pressure group, Bland-Altman plot showed no significant AHI difference between the two groups, the AHI bias is $-0.81 \pm 9.42$ with $95 \%$ limits of agreement $(-19.26$ 17.64). 
Table 2 Intraclass correlation coefficient of AHI results between PSG and radar

\begin{tabular}{lcccccc}
\hline PSG severity & PSG AHI (mean) & Radar AHI (mean) PSG group (n) & $\begin{array}{c}\text { Radar } \\
\text { group (n) }\end{array}$ & $\begin{array}{c}\text { Intraclass } \\
\text { correlation }\end{array}$ & $\begin{array}{c}95 \% \text { confidence } \\
\text { interval }\end{array}$ \\
\hline Normal & 2.32 & 2.75 & 63 & 63 & 0.52 & $0.21-0.71$ \\
Mild & 11.34 & 9.50 & 26 & 25 & 0.49 & $-0.12-0.77$ \\
Moderate & 22.11 & 20.8 & 32 & 34 & 0.70 & 0.047 \\
Severe & 54.93 & 53.75 & 55 & 54 & 0.91 & 0.85 \\
Total & 23.69 & 22.66 & 176 & 176 & 0.98 & $0.84-0.95$ \\
\hline
\end{tabular}

AHI, apnea-hypopnea index; PSG, polysomnography.

Table 3 Sensitivity and specificity of radar AHI compared with PSG AHI

\begin{tabular}{lcccc}
\hline AHI cut-off value & Sensitivity & Specificity & PPV (\%) & NPV (\%) \\
\hline 5 & 1 & 1 & 100 & 100 \\
15 & 0.97 & 0.96 & 98.9 & 100 \\
30 & 0.95 & 0.99 & 98.21 & 98.37 \\
\hline
\end{tabular}

AHI, apnea-hypopnea index; PSG, polysomnography; PPV, positive predictive value; NPV, negative predictive value.

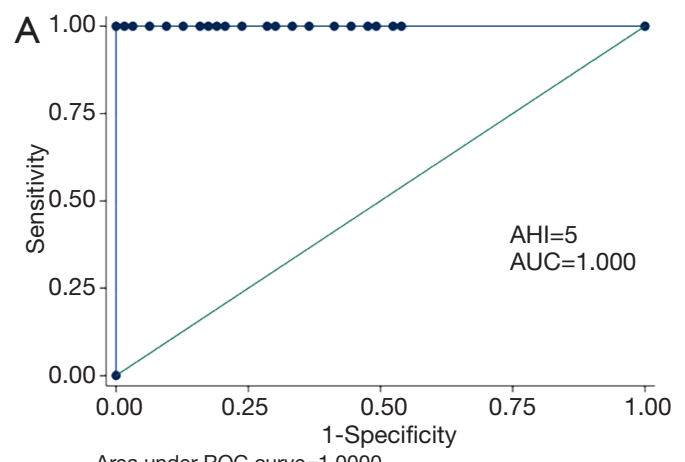

Area under ROC curve $=1.0000$

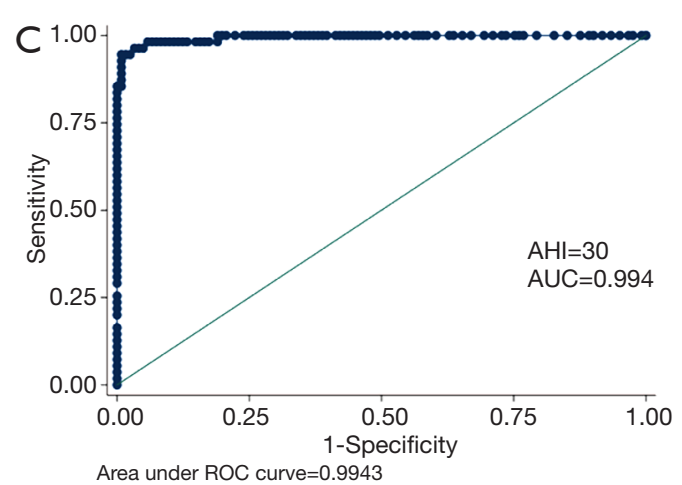

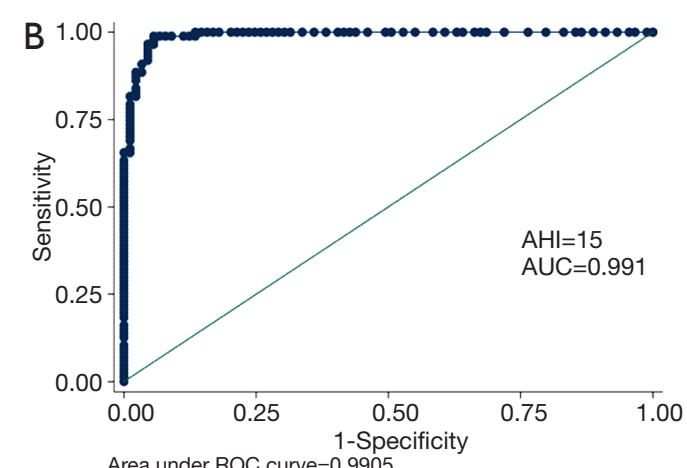

Area under ROC curve $=0.9905$

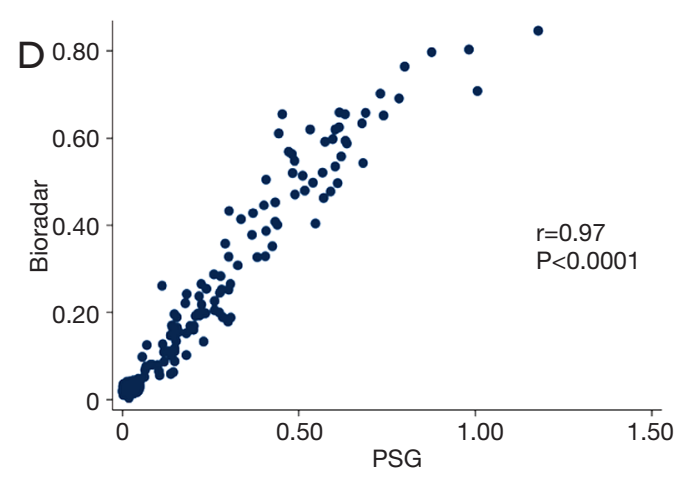

Figure 3 Correlation of wireless UWB radar-AHI and PSG-AHI. (A) ROC curve analysis with a cut-off value of AHI =5, area under curve $1.00 \pm 0.00$, 95\% confidence interval: $1.00-1.00$; (B) ROC curve analysis with cut-off value of AHI $=15$, area under curve $0.99 \pm 0.005,95 \%$ confidence interval: 0.98-1.00; (C) ROC curve analysis with cut-off value of AHI =30, area under curve 0.99 $\pm 0.004,95 \%$ confidence interval: 0.99-1.00; (D) significant correlation was detected between wireless UWB radar sleep screening device-AHI and PSG-AHI (Pearson's r $=0.97,95 \%$ confidence interval: 0.96-0.98, P<0.0001, n=176). UWB, ultra-wideband; AHI, apnea-hypopnea index; PSG, polysomnography. 

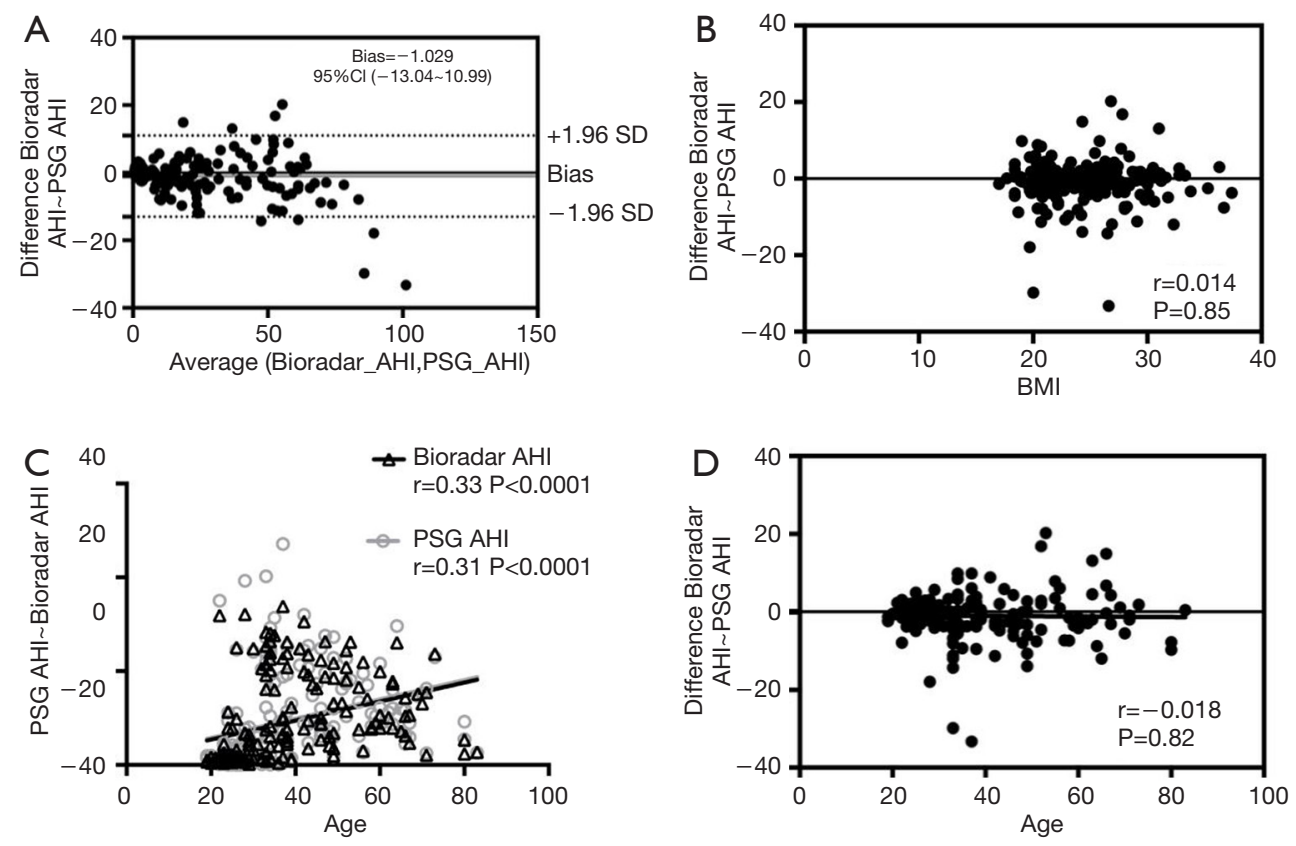

Figure 4 Radar AHI and PSG AHI showed high agreement despite severity and age variations. (A) Radar AHI/PSG AHI agreement was estimated according to Bland-Altman comparison; (B) correlation between BMI and Radar AHI/PSG AHI agreement; (C) correlation between age and Radar AHI/PSG AHI values; (D) correlation between age and Radar AHI/PSG AHI agreement. AHI, apnea-hypopnea index; PSG, polysomnography.

Table 4 Severity of AHI concordance between PSG and radar results ( $\mathrm{n}=176)$

\begin{tabular}{lccccc}
\hline \multirow{2}{*}{ Severity according to PSG } & \multicolumn{4}{c}{ Severity according to radar } \\
\cline { 2 - 5 } & Normal $(\mathrm{AHI}<5)$ & Mild $(5 \leq \mathrm{AHI}<15)$ & Moderate $(\mathrm{AHI} \geq 30)$ & Severe $(\mathrm{AHI} \geq 30)$ \\
\hline Normal $(\mathrm{AHI}<5)$ & 63 & 0 & 0 & 0 & 63 \\
Mild $(5 \leq \mathrm{AHI}<15)$ & 0 & 25 & 1 & 0 & 26 \\
Moderate $(15 \leq \mathrm{AHI}<30)$ & 0 & 0 & 31 & 2 & 53 \\
Severe $(\mathrm{AHI} \geq 30)$ & 0 & 0 & 34 & 54 \\
Total & 63 & 25 & 25 & 176 \\
\hline
\end{tabular}

AHI, apnea-hypopnea index; PSG, polysomnography.

\section{Discussion}

This study aimed to validate a novel, portable wireless UWB radar sleep screening device in obstructive sleep apnea. The UWB radar can detect signals indicating respiratory movement and body motion, count the patient's sleep events and frequency, and adjust the total sleep time. Since no attached sensors on the patient are needed, the portable radar device can simultaneously work with PSG and is non-contact, regardless of the bed mattress and sleep posture. All those features combine to make the device suitable for sleep screening both in the hospital or at home.
Given the high cost and long waitlist of classic standard polysomnography, many types of portable home sleep test devices have recently emerged. Evidence suggests that such devices are a reasonable substitute for in-laboratory polysomnography in most patients, except for some patients with severe cardiopulmonary or neuromuscular diseases (18). Sleep test devices were classified by the American Academy of Sleep Medicine into four levels based on the type of leads and settings. Advanced technology has resulted in a variety of portable monitoring devices that are easy to connect even without attendance of technicians, and can be used at home for obstructive sleep apnea screening or even diagnosis. 

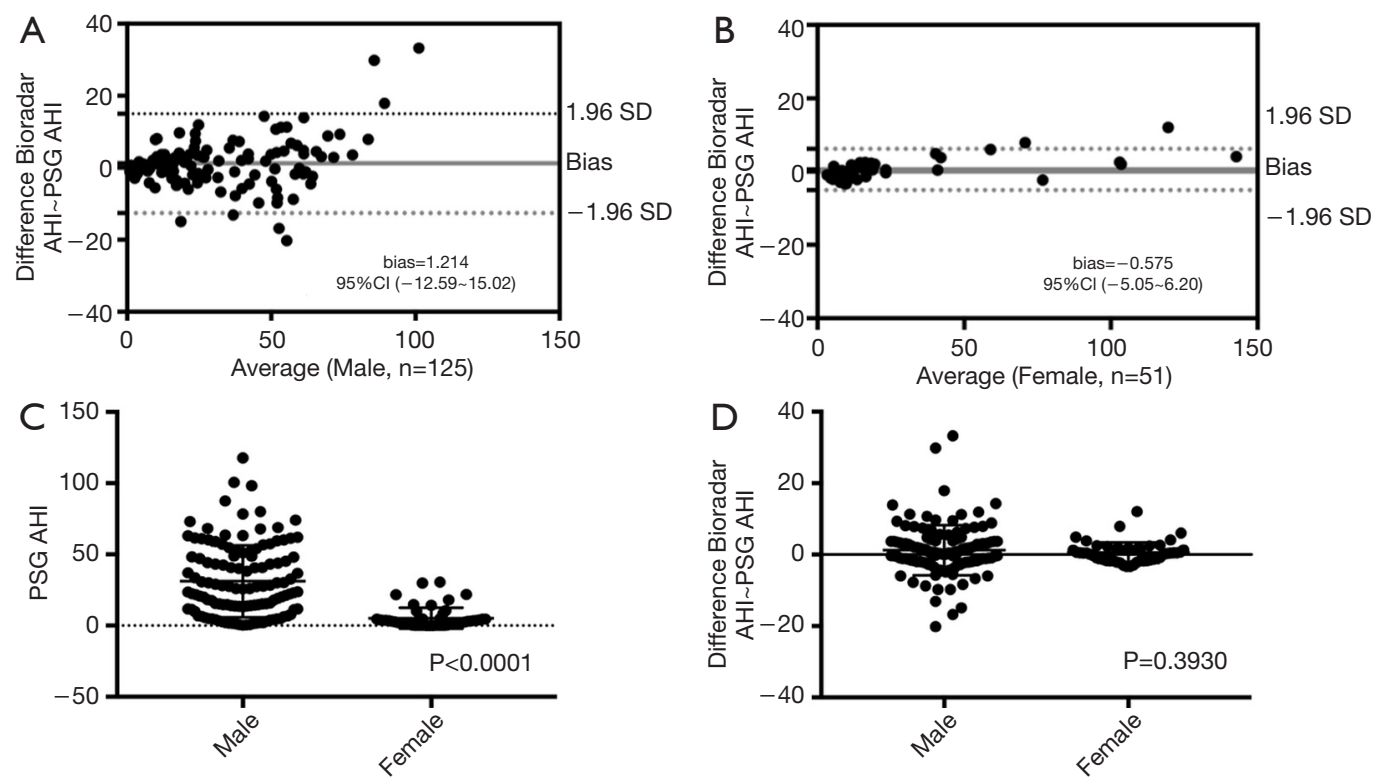

Figure 5 Radar AHI and PSG AHI exhibited high agreement for both male and female participants. (A) Radar AHI/PSG AHI agreement in male participants was estimated according to Bland-Altman plot; (B) radar AHI/PSG AHI agreement in female participants was estimated according to Bland-Altman plot; (C) PSG AHI was higher in male participants than in female counterparts (mean difference $-25.93,95 \%$ confidence interval: -30.83--21.03, P<0.0001); (D) radar AHI/PSG AHI agreement between male and female participants showed no statistical difference (mean difference $-0.64,95 \%$ confidence interval: $-2.12-0.84, \mathrm{P}=0.39$ ). AHI, apnea-hypopnea index; PSG, polysomnography.

Level 2 sleep test devices have more than seven channels and are used at home to increase accessibility and decrease costs. Nevertheless, the proper use of corresponding electrode cables and wires limits unattended home use. However, portable devices simplify these settings at the cost of amounts of data for accurate diagnosis. Thus, the paradox between channel numbers and diagnostic data for unattended devices urge creation of breakthrough technologies.

Systemic review confirmed that level 3 portable devices showed good diagnostic performance in adult patients with a high pre-test probability of moderate to severe OSA with no unstable comorbidities (19). For most patients with suspected OSA, including mild OSA patients, home respiratory polygraphy is similarly effective to PSG (20). The accuracy of level 4 portable devices, on the other hand, merits more investigation. These devices adopt overnight ECG recordings, pulse oxygen saturation, or thoracoabdominal motion detection $(21,22)$. Other devices with mattress- and sheet-like technology can diminish wires, increasing the speed of setup; however, size and cost limit widespread monitoring in homes $(23,24)$. Most of these devices are contact in nature and may generate hygiene or comfort problems. Breath sound detection with microphones has been reported with acceptable accuracy and is easy to apply at home, but many such technologies are snore-dependent and may be susceptible to surrounding acoustic noise such as from other patients in the same general ward, limiting generalization (25). Remote sensing devices with digital camera- and vision-based technology are non-contact and unobtrusive, but are susceptible to movement artifacts and obscurity of patients with cover sheets $(26,27)$.

Radar-based devices are non-contact and patients can use bedding articles freely, but those are still in early experimental stages. In 2002, the Federal Communications Commission (FCC) granted permission for the marketing and operation of a new class of products incorporating ultra-wideband (UWB) technology. The main advantages of UWB radar for real-life implementation of home healthcare application include: high material-penetration capability, low electromagnetic interference and specific absorption rates, good immunity against multipath interference, thereby enhancing measurement reliability, low energy consumption, and diminished size of devices (28). Importantly, costs are dramatically reduced. In 2006, Chen 
first reported respiration rate estimation algorithms with the UWB radar (28); Baboli also proposed a new algorithm that detected heartbeat and respiration rate, while dramatically diminishing interfering environmental motions (29). The algorithm was also able to acquire respiratory features such as the inspiration and expiration speed, respiration intensity, and respiration holding ratio (30). In 2014, Lazaro first reported the application of UWB radar technology in sleeprelated breathing disorders on one volunteer (31), and in 2016, Javaid combined the UWB radar and microphone data to detect sleep apnea events, comparing results with PSG data on three subjects, yielding a sensitivity and specificity of $80 \%$ and $70 \%$, respectively (32).

Our study aimed to validate the accuracy of UWB radar detection in sleep-related breathing disorders. We found a statistically high correlation between UWB radar AHI and PSG AHI.ROC analysis with three different cutoff values revealed satisfying diagnostic efficacy, and high sensitivity and specificity were reached in varying AHI severity groups. Respiratory movement varies with sex and age, and our device can diminish these differences to obtain high concordance with PSG AHI. In addition, UWB radar is non-contact and can be easily delivered via mail or express services, primed for easy installation at home for continuous monitoring without technician attendance. These home applications merit further research, as this unobtrusive device might also benefit pediatric patients or patients with burn injuries. Limitations of this study include the fact that fewer female participants were recruited, which is commonly seen in most sleep disorder studies. Since this device does not include electroencephalogram, it theoretically can't record micro arousals. The thoracoabdominal movement was detected based on physical stretch of bands. It was reported that assessment of the thoracoabdominal movement can indirectly detect respiratory effort-related arousal (33), and the UWB radar, which is based on radar signal, is more sensitive to detect the thoracoabdominal movement, so it can more accurately record the respiratory effort-related arousal. Although the UWB radar does not need the measurement of $\mathrm{SpO}_{2}$, lack of oximetry is the second limitation. In this study, a small group of healthy participants were recruited to verified the cutoff value of AHI =5, and influenced the mean Epworth Sleepiness Scale. Further examinations outside the sleep center are required to validate the radar device, and adding additional channels such as pulse oximetry or heart rate may increase device accuracy and diagnostic value.

\section{Conclusions}

This study suggested that the UWB radar AHI demonstrated an outstanding consistency with PSG AHI. The technology's high sensitivity and specificity meet the competency of obstructive sleep apnea screening or following dynamic monitoring at home, and may be a portable, convenient, and reliable device to screen patients suspected of obstructive sleep apnea. Integrations of big data and artificial intelligence technologies might be promising for this automatic sleep screening device in the near future.

\section{Acknowledgments}

Thanks to Jianjun Wang's assisting in development and maintenance of the UWB radar.

Funding: This work was supported by the Health and Family Planning Commission of Zhejiang Province (Grant number 2018248087 and 2016150952), and the Science and Technology Bureau of Wuyi County.

\section{Footnote}

Conflicts of Interest: All authors have completed the ICMJE uniform disclosure form (available at http://dx.doi. org/10.21037/jtd.2020.02.59). The authors have no conflicts of interest to declare.

Ethical Statement: The authors are accountable for all aspects of the work in ensuring that questions related to the accuracy or integrity of any part of the work are appropriately investigated and resolved. The protocol of our study was approved by the Ethics Committee of Sir Run Run Shaw Hospital, and the research was performed in accordance with relevant guidelines. Informed consent for study participation and publication of identifying information/images in an online open-access publication was obtained from all participants.

Open Access Statement: This is an Open Access article distributed in accordance with the Creative Commons Attribution-NonCommercial-NoDerivs 4.0 International License (CC BY-NC-ND 4.0), which permits the noncommercial replication and distribution of the article with the strict proviso that no changes or edits are made and the original work is properly cited (including links to both the 
formal publication through the relevant DOI and the license). See: https://creativecommons.org/licenses/by-nc-nd/4.0/.

\section{References}

1. Franklin KA, Lindberg E. Obstructive sleep apnea is a common disorder in the population-a review on the epidemiology of sleep apnea. J Thorac Dis 2015;7:1311-22.

2. Peppard PE, Hagen EW. The Last 25 Years of Obstructive Sleep Apnea Epidemiology-and the Next 25? Am J Respir Crit Care Med 2018;197:310-2.

3. Lattanzi S, Brigo F, Silvestrini M. Blood pressure profile and nocturnal oxygen desaturation. J Clin Hypertens (Greenwich) 2018;20:656-8.

4. Lattanzi S, Brigo F, Silvestrini M. Obstructive sleep apnea syndrome and the nocturnal blood pressure profile. J Clin Hypertens (Greenwich) 2018;20:1036-8.

5. Stevens SL, Wood S, Koshiaris C, et al. Blood pressure variability and cardiovascular disease: systematic review and meta-analysis. BMJ 2016;354:14098.

6. Lattanzi S, Vernieri F, Silvestrini M. Blood pressure variability and neurocognitive functioning. J Clin Hypertens (Greenwich) 2018;20:645-7.

7. Tully PJ, Yano Y, Launer LJ, et al. Association Between Blood Pressure Variability and Cerebral Small-Vessel Disease: A Systematic Review and Meta-Analysis. J Am Heart Assoc 2020;9:e013841.

8. Jonas DE, Amick HR, Feltner C, et al. Screening for Obstructive Sleep Apnea in Adults: Evidence Report and Systematic Review for the US Preventive Services Task Force. JAMA 2017;317:415-33.

9. Takizawa K, Li HB, Hamaguchi K, et al. Wireless vital sign monitoring using ultra wideband-based personal area networks. Conf Proc IEEE Eng Med Biol Soc 2007;2007:1798-801.

10. Kagawa M, Tojima H, Matsui T. Non-contact diagnostic system for sleep apnea-hypopnea syndrome based on amplitude and phase analysis of thoracic and abdominal Doppler radars. Med Biol Eng Comput 2016;54:789-98.

11. De Chazal P, Fox N, O'Hare E, et al. Sleep/wake measurement using a non-contact biomotion sensor. J Sleep Res 2011;20:356-66.

12. Amir O, Barak-Shinar D, Henry A, et al. Photoplethysmography as a single source for analysis of sleep-disordered breathing in patients with severe cardiovascular disease. J Sleep Res 2012;21:94-100.

13. Beattie ZT, Hayes TL, Guilleminault C, et al. Accurate scoring of the apnea-hypopnea index using a simple non- contact breathing sensor. J Sleep Res 2013;22:356-62.

14. Bianchi MT, Lipoma T, Darling C, et al. Automated sleep apnea quantification based on respiratory movement. Int J Med Sci 2014;11:796-802.

15. Zeng T, Mott C, Mollicone D, et al. Automated determination of wakefulness and sleep in rats based on non-invasively acquired measures of movement and respiratory activity. J Neurosci Methods 2012;204:276-87.

16. de Zambotti M, Baker FC, Colrain IM. Validation of Sleep-Tracking Technology Compared with Polysomnography in Adolescents. Sleep 2015;38:1461-8.

17. Van Nguyen, Javaid AQ, Weitnauer MA. Spectrumaveraged Harmonic Path (SHAPA) algorithm for noncontact vital sign monitoring with ultra-wideband (UWB) radar. Conf Proc IEEE Eng Med Biol Soc 2014;2014:2241-4.

18. Cooksey JA, Balachandran JS. Portable Monitoring for the Diagnosis of OSA. Chest 2016;149:1074-81.

19. El Shayeb M, Topfer LA, Stafinski T, et al. Diagnostic accuracy of level 3 portable sleep tests versus level 1 polysomnography for sleep-disordered breathing: a systematic review and meta-analysis. CMAJ 2014;186:E25-51.

20. Corral J, Sanchez-Quiroga MA, Carmona-Bernal C, et al. Conventional Polysomnography Is Not Necessary for the Management of Most Patients with Suspected Obstructive Sleep Apnea. Noninferiority, Randomized Controlled Trial. Am J Respir Crit Care Med 2017;196:1181-90.

21. de Chazal P, Heneghan C, Sheridan E, et al. Automated processing of the single-lead electrocardiogram for the detection of obstructive sleep apnoea. IEEE Trans Biomed Eng 2003;50:686-96.

22. Gyulay S, Gould D, Sawyer B, et al. Evaluation of a microprocessor-based portable home monitoring system to measure breathing during sleep. Sleep 1987;10:130-42.

23. Polo O, Brissaud L, Sales B, et al. The validity of the static charge sensitive bed in detecting obstructive sleep apnoeas. Eur Respir J 1988;1:330-6.

24. Tenhunen M, Elomaa E, Sistonen H, et al. Emfit movement sensor in evaluating nocturnal breathing. Respir Physiol Neurobiol 2013;187:183-9.

25. Ben-Israel N, Tarasiuk A, Zigel Y. Nocturnal sound analysis for the diagnosis of obstructive sleep apnea. Conf Proc IEEE Eng Med Biol Soc 2010;2010:6146-9.

26. McDuff D, Gontarek S, Picard RW. Improvements in remote cardiopulmonary measurement using a five band digital camera. IEEE Trans Biomed Eng 2014;61:2593-601.

27. Li MH, Yadollahi A, Taati B. A non-contact vision-based 
system for respiratory rate estimation. Conf Proc IEEE

Eng Med Biol Soc 2014;2014:2119-22.

28. Chen Y, Gunawan E, Low KS, et al. Non-invasive respiration rate estimation using ultra-wideband distributed cognitive radar system. Conf Proc IEEE Eng Med Biol Soc 2006;2006:920-3.

29. Baboli M, Boric-Lubecke O, Lubecke V. A new algorithm for detection of heart and respiration rate with UWB signals. Conf Proc IEEE Eng Med Biol Soc 2012;2012:3947-50.

30. Hsieh CH, Chiu YF, Shen YH, et al. A UWB Radar Signal Processing Platform for Real-Time Human Respiratory Feature Extraction Based on Four-Segment Linear

Cite this article as: Zhou Y, Shu D, Xu H, Qiu Y, Zhou P, Ruan W, Qin G, Jin J, Zhu H, Ying K, Zhang W, Chen E. Validation of novel automatic ultra-wideband radar for sleep apnea detection. J Thorac Dis 2020;12(4):1286-1295. doi: 10.21037/ jtd.2020.02.59
Waveform Model. IEEE Trans Biomed Circuits Syst 2016;10:219-30.

31. Lazaro A, Girbau D, Villarino R. Techniques for clutter suppression in the presence of body movements during the detection of respiratory activity through UWB radars. Sensors (Basel) 2014;14:2595-618.

32. Javaid AQ, Noble CM, Rosenberg R, et al. Towards detection of sleep apnea events by combining different non-contact measurement modalities. Conf Proc IEEE Eng Med Biol Soc 2016;2016:5307-10.

33. Masa JF, Corral J, Martin MJ, et al. Assessment of thoracoabdominal bands to detect respiratory effortrelated arousal. Eur Respir J 2003;22:661-7. 